\title{
Total Excision of Tongue
}

National Cancer Institute

\section{Source}

National Cancer Institute. Total Excision of Tongue. NCI Thesaurus. Code C51598.

Surgery involving complete excision of the tongue. 